ensure participant safety. It was this analysis by the Data Safety Monitoring Board that sparked the concern about safety with the adenovirus vector. The trial has certainly raised questions about whether the induction of HIV-specific T-cell responses is a viable vaccine strategy, but these questions are best addressed by analysing the data.

Particularly important are questions regarding the effect of the quality, quantity and specificity of the vaccine-induced CD8 T-cell response on postinfection viral load control. These questions could not be addressed without a vaccine approach that actually induced CD8 T-cell responses in most recipients.

The Merck vaccine was the first such candidate, so it is a misleading exaggeration to claim that its failure is a "crisis" for HIV vaccine research. A journal with Nature's long history is well placed to know how likely first-time successes are in science.

In my view, your Editorial risks reinforcing the unrealistic expectations of science that you erroneously imply were promoted by the HIV-vaccine researchers involved in the Merck trial.

Richard Jefferys Michael Palm Basic Science, Vaccines \& Prevention Project, Treatment Action Group, 611 Broadway, Suite 308, New York, New York 100012, USA

\section{Fixing hiring practices means asking the right question}

SIR - Whenever Spain's research or academic life is analysed (see, for example, Nature 451, 1029; 2008), a comment invariably follows on hiring practices and regulations (Nature 453, 26-27; 2008). This obsessive repetition points to a deep problem in that country, but these issues are shared by many other nations and so deserve international attention.

The question that is usually asked or answered concerns 'how': how should the academic system be regulated to avoid widespread poor practices in hiring? As an academic, I would start by asking 'why': why is it that the hiring decisions of academics in some countries cannot be trusted and apparently need to be enforced by tight regulations, whereas this is not the case in other countries?

Remedies don't work well without a good diagnostic. Emilio Artacho Department of Earth Sciences, University of Cambridge, Downing Street, Cambridge CB2 3EQ, UK

\section{Germline modification carries risk of major social harm}

SIR - Your Editorial 'New sources of sex cells' (Nature 452, 913;

2008), on the potential use of pluripotent stem-cell-derived gametes (PSCDGs) for germline genetic modification and enhancement, suggests that the prospect of stem-cell-derived gametes could trigger renewed calls for regulating human biotechnologies. In those discussions we must, as you warn, be wary of impeding basic research. But we must be equally willing to draw lines proscribing socially pernicious applications.

Germline (that is, inheritable) modification is the most socially consequential and ethically dubious application of human biotechnology; its implications have been explored from a wide range of perspectives. Most of these discussions have focused on the social meaning and repercussions of genetic manipulation of the human species, not on the moral status of human embryos. One can strongly support human-embryo research and still oppose germline modification. Conversely, some opponents of abortion rights support germline modification, so long as no embryos are destroyed in the process.

Although there may be scientific and therapeutic benefits from research on PSCDGs, the case for any such benefits from using these cells for human germline modification is weak, whereas the likelihood of substantial harm is great. One consequence could be inequalities between those who can afford genetic enhancements and the majority who cannot. Human-germline modification could lead to the emergence of 'genetic castes', creating vast social rifts, with horrific consequences. Such considerations have already prompted many countries including most of Europe, Canada, Japan, South Africa and Brazil - to prohibit germline modification.

Marcy Darnovsky Center for Genetics and Society, 436 14th Street, Suite 700, Oakland, California 94612, USA

\section{Global database is needed to support adaptation science}

SIR - Cynthia Rosenzweig and colleagues have taken a critical step towards a global synthesis of biological and physical impacts attributable to climate change (Nature 453, 353-357; 2008).

They have expanded the database used in the recent Fourth

Assessment from the

Intergovernmental Panel on

Climate Change (IPCC). We feel

there are still many more studies

that could be compiled to improve geographical and ecosystem imbalances and to provide a more comprehensive overview. Given the resources and effort required to identify these, it is beyond the scope of a limited number of individuals.

Climate-change impacts are being reported in a burgeoning literature from every ocean and continent. It is now time to shift the emphasis from proving climate impacts to providing key support for adaptation science. This requires a publicly accessible global database to collate research into climate-change impacts research and to allow the scientific community and the IPCC to focus additional efforts on attribution and adaptation.
Global cooperation and sharing model outputs have led to breakthroughs in understanding the climate system, as illustrated by the scientific community's assembling of the physicalscience basis (IPCC working group I). We suggest the impacts community (working group II) should follow a similar path.

Just as the IPCC endorsed the Program for Climate Model Diagnosis and Intercomparison data repository for model projections, so could it endorse an impacts repository, with researchers able continuously to upload research results that can then be quality-assured by a verification panel. The panel could be selected on the basis of expertise across a range of disciplines, and the repository website hosted by an international body, such as the International Geosphere-Biosphere Programme. Similar global initiatives underline the value of such an approach. These include the Ocean Biogeographic Information System (set up by the Census of Marine Life) and GenBank (National Center for Biotechnology).

This approach would allow greater efficiency, transparency and completeness in the compilation process, facilitate rapid identification of knowledge gaps and allow broader, expertdriven quality control of the interpretation of biological, aquatic and terrestrial data. This pathway to transparency and rigorous global synthesis is critical for a potential Fifth Assessment Report and for a world relying on scientific guidance.

Elvira S. Poloczanska, Alistair

J. Hobday Climate Adaptations Flagship, Commonwealth Scientific and Industrial Research Organisation (CSIRO), Marine and Atmospheric Research, GPO Box 1538, Hobart, Tasmania 7001, Australia Anthony J. Richardson Climate Adaptations Flagship, CSIRO, Marine and Atmospheric Research, PO Box 120, Cleveland, Queensland 4163, Australia and University of Queensland, Department of Mathematics, St Lucia, Queensland 4072, Australia 